\title{
Order polynomials and Pólya's enumeration theorem
}

\author{
Katharina Jochemko* \\ Fachbereich Mathematik und Informatik \\ Freie Universität Berlin \\ Berlin, Germany \\ jochemko@math.fu-berlin.de
}

Submitted: Feb 2, 2014; Accepted: Jun 12, 2014; Published: Jun 27, 2014

Mathematics Subject Classifications: 06A07, 06A11, 05A19 , 05C15, 05C31, 05E18

\begin{abstract}
Pólya's enumeration theorem states that the number of labelings of a finite set up to symmetry is given by a polynomial in the number of labels. We give a new perspective on this theorem by generalizing it to partially ordered sets and order preserving maps. Further we prove a reciprocity statement in terms of strictly order preserving maps generalizing a classical result by Stanley (1970). We apply our results to counting graph colorings up to symmetry.
\end{abstract}

\section{Introduction}

Counting objects up to symmetry is a basic problem of enumerative combinatorics. A fundamental result in this context is Pólya's enumeration theorem which is concerned with counting labelings of a set of objects modulo symmetry. Here a labeling of a set $X$ is defined as a map $f: X \rightarrow Y$ where $Y$ is the set of labels. If $G$ is a group acting on $X$ then $G$ also acts on the set of labelings $Y^{X}:=\{f: X \rightarrow Y\}$. Pólya's enumeration theorem now states:

Theorem 1.1 (Pólya's enumeration theorem [3]). Let $G$ be a finite group acting on a finite set $X$ and let $Y$ be a finite set of $n=|Y|$ labels. Then

$$
\left|Y^{X} / G\right|=\frac{1}{|G|} \sum_{g \in G} n^{c(g)}
$$

where $Y^{X} / G$ is the collection of orbits of $Y^{X}$ and $c(g)$ is the number of cycles of $g$ as permutation of $X$.

\footnotetext{
*Supported by a Hilda Geiringer Scholarship at the Berlin Mathematical School.
} 
We give a new perspective on this theorem by generalizing it in terms of partially ordered sets, or posets for short, and order preserving maps. More precisely, we consider a finite poset $P$ and a group $G$ acting on $P$ by automorphisms. Then $G$ acts in a natural way also on the set of all order preserving maps $\operatorname{Hom}(P,[n])$ from $P$ into the $n$-chain $[n]=\{1<\cdots<n\}$. We show that the number of orbits of $\operatorname{Hom}(P,[n])$ is given by a polynomial $\Omega_{P, G}(n)$ which we call the orbital order polynomial. Pólya's enumeration theorem then follows by specializing this result to antichains. Further we give a combinatorial interpretation for $\Omega_{P, G}(-n)$ in terms of orbits of strictly order preserving maps. This naturally generalizes the classical polynomiality and reciprocity theorems for order preserving maps due to Stanley [4]. These results can be furthermore generalized to counting $(P, \omega)$-partitions up to symmetry.

The results can be applied to graph colorings. We consider a finite group $G$ acting by automorphisms on a finite simple graph $\Gamma=(V, E)$ and the function $\chi_{\Gamma, G}(n)$ counting proper colorings $c: V \rightarrow[n]$ up to group action. Cameron and Kayibi [1] seem to be the first who considered this function which they called the orbital chromatic polynomial. Previously, Hanlon [2] treated the case of $G$ being the automorphism group of $\Gamma$. It is easy to see that $\chi_{\Gamma, G}(n)$ indeed agrees with a polynomial for all $n \geqslant 1$. We further give a representation as a sum of order polynomials.

We also give a combinatorial interpretation for evaluating this polynomial at negative integers in terms of acyclic orientations and compatible colorings. This naturally generalizes Stanley's reciprocity for graph colorings [6].

\section{Order preserving Pólya-enumeration}

\subsection{Groups}

Let $G$ be a finite group with identity element $e$ and $X$ be a finite set. A group operation of $G$ on $X$ is a map $: G \times X \rightarrow X$ such that $g \cdot(h \cdot x)=(g h) \cdot x$ and $e \cdot x=x$ for all $g, h \in G$ and $x \in X$. We say that $G$ operates or acts on $X$. For every $g \in G$ we denote by $X^{g}$ the fixpoints of $g$, i.e., $X^{g}=\{x \in X: g \cdot x=x\}$. For an element $x \in X$ we denote by $G x=\{g \cdot x: g \in G\}$ the orbit of $x$. The set of all orbits partitions $X$ and is called $X / G$.

Burnside's lemma (see e.g. [9, Theorem 10.5]) gives a formula for the number of orbits in terms of fixpoints:

Theorem 2.1 (Burnside's lemma). Let $G$ be a finite group acting on a finite set $X$. Then

$$
|X / G|=\frac{1}{|G|} \sum_{g \in G}\left|X^{g}\right| .
$$

For an element $x \in X$ the stabilizer of $x$ is $\operatorname{Stab}(x)=\{g \in G: g \cdot x=x\}$. The operation can be restricted to any subgroup $H \subseteq G$. For $g \in G$ we denote by $\langle g\rangle=\left\{e, g, g^{2}, \ldots\right\}$ the cyclic subgroup generated by $g$. The orbit of $x$ under the action of $\langle g\rangle$ is denoted by 
$[x]_{g}$ and is called a cycle of $g$. In particular, $c(g)=|X /\langle g\rangle|$ is the number of cycles of $g$. Identifying $g$ with the corresponding permutation on $X$ gives the usual notion of cycles.

Example 2.2. Let $G=S_{n}$ be the symmetric group acting on $[n]=\{1, \ldots, n\}$ in the usual way. Every permutation $\sigma \in S_{n}$ can be written as product of disjoint cycles in $S_{n}$ and this representation is unique up to interchanging the order of the cycles in the product. In this case $[x]_{\sigma}=\{y \in[n]: y$ and $x$ are in the same cycle $\}$ and $c(\sigma)$ is the number of cycles in the unique representation as product of disjoint cycles.

If a set $X$ has additional structure we say that a group $G$ acts by automorphisms on $X$ if the group operation respects the structure, that is, for all $g \in G$ the map $x \mapsto g \cdot x$ is a structure preserving bijection.

Let $P$ be a finite poset. Then a group $G$ acts on $P$ by automorphisms if for all $g \in G$ and for all $p$ and $q$ in $P$ we have $g \cdot p \prec g \cdot q$ whenever $p \prec q$.

For a finite simple graph $\Gamma=(V, E)$ an action of a group $G$ on $V$ respects the structure of $\Gamma$ if for all edges $u v \in E$ we have that there is an edge between $g \cdot u$ and $g \cdot v$ for all $g \in G$.

The operation of $G$ on $X$ induces an operation on $Y^{X}$. This induced operation is defined by $(g \cdot f)(x)=f\left(g^{-1} x\right)$ for all $g \in G, f \in Y^{X}$ and $x \in X$.

\section{$2.2 \quad$ Order preserving maps}

Let $P$ be a finite poset. A map $\phi: P \rightarrow[n]$ is called order preserving if $\phi(p) \leqslant \phi(q)$ whenever $p \prec q$ and equality is forbidden for strictly order preserving maps. We denote the set of all order preserving maps by $\operatorname{Hom}(P,[n])$, and the set of strictly order preserving maps by $\operatorname{Hom}^{\circ}(P,[n])$. Their cardinalities are given by the order polynomials $\Omega_{P}(n)$ and $\Omega_{P}^{\circ}(n)$ respectively. The following classical result is due to Stanley [4].

Theorem 2.3 (Stanley [4]). For a finite poset $P$ the function $\Omega_{P}(n)$ agrees with a polynomial of degree $|P|$ for all $n \geqslant 1$, and

$$
\Omega_{P}^{\circ}(n)=(-1)^{|P|} \Omega_{P}(-n) .
$$

For every finite group $G$ acting on $P$ by automorphisms we define a partial order on $P / G$ by defining $G x \prec G y$ whenever there are $\tilde{x} \in G x$ and $\tilde{y} \in G y$ such that $\tilde{x} \prec \tilde{y}$.

This, in fact, yields a poset, the quotient poset (see e.g. [7]).

Lemma 2.4. Let $P$ be a finite poset and $G$ a finite group acting by automorphisms on $P$. Then $P / G$ is a poset.

Proof. For irreflexivity we observe that every orbit $G x$ is an antichain. For that suppose $g x \prec h x$ for some $g, h \in G$ and consequently $x \prec g^{-1} h x$. Then it follows that $x \prec g^{-1} h x \prec\left(g^{-1} h\right)^{2} x \prec \cdots \prec x$ as $g^{-1} h$ has finite order which is a contradiction as $P$ is a poset.

For transitivity let $\tilde{x} \in G x, \tilde{y}, \bar{y} \in G y$ and $\bar{z} \in G z$ with $\tilde{x} \prec \tilde{y}$ and $\bar{y} \prec \bar{z}$. Then there exists a $g \in G$ with $g \tilde{y}=\bar{y}$ and we have $g \tilde{x} \prec g \tilde{y}=\bar{y} \prec \bar{z}$. 
For $g \in G$ we define $P_{g}=P /\langle g\rangle$. Then $P_{g}$ is a poset with $c(g)$ elements.

An order preserving action of $G$ on $P$ induces an action on $\operatorname{Hom}(P,[n])$ and $\operatorname{Hom}^{\circ}(P,[n])$ as subsets of $[n]^{P}$. We define the orbital order polynomials $\Omega_{P, G}(n)=|\operatorname{Hom}(P,[n]) / G|$ and $\Omega_{P, G}^{\circ}(n)=\left|\operatorname{Hom}^{\circ}(P,[n]) / G\right|$ for $n \geqslant 1$. The following main theorem states that $\Omega_{P, G}(n)$ and $\Omega_{P, G}^{\circ}(n)$ are indeed polynomials for $n \geqslant 1$, and gives formulas in terms of order polynomials:

Theorem 2.5. Let $G$ be a finite group acting by automorphisms on a finite poset $P$. Then

$$
\begin{aligned}
& \Omega_{P, G}(n)=\frac{1}{|G|} \sum_{g \in G} \Omega_{P_{g}}(n), \\
& \Omega_{P, G}^{\circ}(n)=\frac{1}{|G|} \sum_{g \in G} \Omega_{P_{g}}^{\circ}(n)
\end{aligned}
$$

for $n \geqslant 1$. In particular, $\Omega_{P, G}(n)$ and $\Omega_{P, G}^{\circ}(n)$ agree with polynomials of degree $|P|$ for $n \geqslant 1$.

Proof. We only show equation (1) as the argument for equation (2) is analoguous. By Theorem 2.1 we have

$$
|\operatorname{Hom}(P,[n]) / G|=\frac{1}{|G|} \sum_{g \in G}\left|\operatorname{Hom}(P,[n])^{g}\right|
$$

By definition, $\phi \in \operatorname{Hom}(P,[n])^{g}$ if and only if $\phi\left(g^{-1} x\right)=\phi(x)$ for all $x \in P$. But this is equivalent to $\phi$ being constant on $[x]_{g}$. Therefore

$$
\begin{aligned}
\operatorname{Hom}(P,[n])^{g} & \rightarrow \operatorname{Hom}\left(P_{g},[n]\right) \\
\varphi & \mapsto\left([x]_{g} \mapsto \varphi(x)\right)
\end{aligned}
$$

is a one-to-one correspondence. Further, observe that $\operatorname{deg} \Omega_{P_{g}}(n)=c(g)=|P|$ if and only if $g$ acts trivially on $P$, and $c(e)=|P|$.

By applying Theorem 2.5 to antichains, we get Pólya's enumeration theorem in the language of posets:

Corollary 2.6. Let $G$ be a finite group acting by automorphisms on a finite antichain $A$, and let $Y=[n]$. Then

$$
\left|Y^{A} / G\right|=\frac{1}{|G|} \sum_{g \in G} n^{c(g)} .
$$

Proof. As $A$ is an antichain we have $\operatorname{Hom}(A,[n])=Y^{A}$. The result follows by observing that $A_{g}$ is an antichain with $c(g)$ elements for all $g \in G$ and $\Omega_{A_{g}}(n)=n^{\left|A_{g}\right|}$.

Example 2.7. Let $S_{k}$ be the symmetric group acting on an antichain $A=\left\{x_{1}, \ldots, x_{k}\right\}$ on $k$ elements by permuting indices, and let $Y=[n]$. Then

$$
\left\{\phi \in Y^{A}: 1 \leqslant \phi\left(x_{1}\right) \leqslant \cdots \leqslant \phi\left(x_{k}\right) \leqslant n\right\}
$$

is a set of representatives of $\operatorname{Hom}(A, Y) / S_{k}$ and therefore

$$
\Omega_{A, S_{k}}(n)=\Omega_{[k]}(n)=\left(\begin{array}{c}
n+k-1 \\
k
\end{array}\right) .
$$




\subsection{Combinatorial reciprocity}

Let $G$ be a finite group acting on a finite poset $P$ by automorphisms. As the number of orbits $|\operatorname{Hom}(P,[n]) / G|$ agrees with a polynomial by Theorem 2.5 we ponder the question if there is a combinatorial interpretation for evaluating this polynomial at negative integers. For that we have to consider a certain class of order preserving maps. The $\operatorname{sign} \operatorname{sgn}(g)$ of an element $g \in G$ is defined as the sign of $g$ as a permutation of $P$ and is equal to $(-1)^{|P|+c(g)}$. An order preserving map $\phi \in \operatorname{Hom}(P,[n])$ is called even if for all $g \in \operatorname{Stab}(\phi)$ we have $\operatorname{sgn}(g)=1$. The set of all even order preserving maps is denoted by $\operatorname{Hom}_{+}(P,[n])$, and we define $\operatorname{Hom}_{+}^{\circ}(P,[n]):=\operatorname{Hom}_{+}(P,[n]) \cap \operatorname{Hom}^{\circ}(P,[n])$ to be the set of even strictly order preserving maps. One observes that the action of $G$ on $\operatorname{Hom}(P,[n])$ restricts to an action on $\operatorname{Hom}_{+}(P,[n])$. For these notions the following reciprocities hold:

Theorem 2.8. Let $G$ be a finite group acting by automorphisms on a finite poset $P$. Then

$$
\begin{aligned}
& \Omega_{P, G}(-n)=(-1)^{|P|}\left|\operatorname{Hom}_{+}^{\circ}(P,[n]) / G\right|, \\
& \Omega_{P, G}^{\circ}(-n)=(-1)^{|P|}\left|\operatorname{Hom}_{+}(P,[n]) / G\right| .
\end{aligned}
$$

Proof. Again, we only show equation (3) as equation (4) follows by analogous arguments. By equation (1) and Theorem 2.3 we have

$$
\Omega_{P, G}(-n)=\frac{1}{|G|} \sum_{g \in G}(-1)^{\left|P_{g}\right|} \Omega_{P_{g}}^{\circ}(n) .
$$

We observe that $\left|P_{g}\right|=c(g)$ is the number of orbits under the action of $\langle g\rangle$. Therefore equation (5) becomes

$$
\begin{aligned}
\Omega_{P, G}(-n) & =(-1)^{|P|} \frac{1}{|G|} \sum_{g \in G} \operatorname{sgn}(g)\left|\operatorname{Hom}^{\circ}(P,[n])^{g}\right| \\
& =(-1)^{|P|} \frac{1}{|G|} \sum_{\phi \in \operatorname{Hom}^{\circ}(P,[n])} \sum_{g \in \operatorname{Stab}(\phi)} \operatorname{sgn}(g) .
\end{aligned}
$$

For $\phi \in \operatorname{Hom}^{\circ}(P,[n])$ and $g_{0} \in \operatorname{Stab}(\varphi)$ such that $\operatorname{sgn}\left(g_{0}\right)=-1$ there is a bijection

$$
\begin{aligned}
\{g \in \operatorname{Stab}(\phi): \operatorname{sgn}(g)=1\} & \longrightarrow\{g \in \operatorname{Stab}(\phi): \operatorname{sgn}(g)=-1\} \\
g & \mapsto g_{0} g
\end{aligned}
$$

Hence, $\sum_{g \in \operatorname{Stab}(\phi)} \operatorname{sgn}(g)=0$ whenever $\phi$ is not even. Therefore the right-hand side of equation (5) equals

$$
(-1)^{|P|} \frac{1}{|G|} \sum_{g \in G}\left|\operatorname{Hom}_{+}^{\circ}(P,[n])^{g}\right|
$$

which equals $(-1)^{|P|}\left|\operatorname{Hom}_{+}^{\circ}(P,[n]) / G\right|$ by Theorem 2.1 .

In the setting of Pólya's enumeration theorem the statement simplifies: 
Corollary 2.9. Let $G$ be a finite group acting on a finite antichain $A$, and let $Y=[n]$. Then

$$
\Omega_{A, G}(-n)=(-1)^{|A|}\left|\operatorname{Hom}_{+}(A,[n]) / G\right| \text {. }
$$

Proof. This follows from the fact that every order preserving map from an antichain is automatically strictly order preserving.

Example 2.10. In Example 2.7 we have $\phi \in \operatorname{Hom}_{+}(A,[n])$ if and only if $\phi$ is injective. Therefore

$$
\left\{\phi \in Y^{A}: 1 \leqslant \phi\left(x_{1}\right)<\cdots<\phi\left(x_{k}\right) \leqslant n\right\}
$$

is a set of representatives for $\operatorname{Hom}_{+}(A,[n]) / S_{k}$. Therefore

$$
\left|\operatorname{Hom}_{+}(A,[n]) / S_{k}\right|=\Omega_{[k]}^{\circ}(n)=\left(\begin{array}{l}
n \\
k
\end{array}\right) .
$$

Remark 2.11. An alternative, geometric route is by way of Ehrhart theory of order polytopes. Geometrically the setting can be translated into counting lattice points in order polytopes where the action of the group is given by permuting coordinates. This complements results by Stapledon [8] who considers lattice preserving group actions and counts lattice points inside stable rational polytopes.

\section{$2.4(P, \omega)$-partitions}

Theorem 2.5 and Theorem 2.8 hold, in fact, in greater generality for $(P, \omega)$-partitions which were first considered by Stanley in [5]. Let $P$ be a poset and let $\omega: P \rightarrow \mathbb{R}$, such that $\omega(p) \neq \omega(q)$ whenever $p$ and $q$ are comparable. An order preserving map $f \in \operatorname{Hom}(P,[n])$ is a $(\boldsymbol{P}, \boldsymbol{\omega})$-partition if for all $p, q \in P$

$$
p \prec q \quad \text { and } \quad \omega(p)>\omega(q) \Longrightarrow f(p)<f(q) .
$$

The pairs $\{(p, q): p \prec q, \omega(p)>\omega(q)\}$ are called inversions. Therefore, a $(P, \omega)$-partition is an order preserving map which is strict on inversions given by $\omega$. Let $\operatorname{Hom}^{\omega}(P,[n])$ be the set of all $(P, \omega)$-partitions $P \rightarrow[n]$. We observe that if $\omega$ is order preserving then $\operatorname{Hom}^{\omega}(P,[n])$ simply equals $\operatorname{Hom}(P,[n])$. If $\omega$ is order reversing then we have $\operatorname{Hom}^{\omega}(P,[n])=\operatorname{Hom}^{\circ}(P,[n])$.

Stanley considered in $[5]$ the $(\boldsymbol{P}, \boldsymbol{\omega})$-polynomial $\Omega_{P}^{\omega}(n)=\left|\operatorname{Hom}^{\omega}(P,[n])\right|$ and showed the following generalization of Theorem 2.3:

Theorem 2.12 (Stanley [5]). Let $P$ be a finite poset and $\omega: P \rightarrow \mathbb{R}$ be a map, such that $\omega(p) \neq \omega(q)$ whenever $p$ and $q$ are comparable. Then $\Omega_{P}^{\omega}(n)$ agrees with a polynomial of degree $|P|$ for $n \geqslant 1$ and

$$
\Omega_{P}^{\omega}(-n)=(-1)^{|P|} \Omega_{P}^{-\omega}(n)
$$


Now let $G$ be a group acting on $P$ by automorphisms which preserve inversions, i.e. for all $g \in G$ we have $\omega(p)<\omega(q) \Leftrightarrow \omega(g p)<\omega(g q)$ for all comparable $p$ and $q$. For $g \in G$ we define $\omega_{g}: P_{g} \rightarrow \mathbb{R}$ by $\omega_{g}\left([x]_{g}\right)=\frac{1}{\langle\langle g\rangle|} \sum_{\tilde{x} \in[x]_{g}} \omega(\tilde{x})$. It is easy to see that $\omega_{g}$ takes different values on comparable elements in $P_{g}$. Analogously as in the case of ordinary order preserving maps we define the orbital $(\boldsymbol{P}, \boldsymbol{\omega})$-polynomial $\Omega_{P, G}^{\omega}(n)=\left|\operatorname{Hom}^{\omega}(P,[n]) / G\right|$, and $\operatorname{Hom}_{+}^{\omega}(P,[n])=\operatorname{Hom}^{\omega}(P,[n]) \cap \operatorname{Hom}_{+}(P,[n])$. By very similar arguments as in the proofs of Theorem 2.5 and Theorem 2.8 we have the following generalization:

Theorem 2.13. Let $P$ be a finite poset and $\omega: P \rightarrow \mathbb{R}$ be a map, such that $\omega(p) \neq \omega(q)$ whenever $p$ and $q$ are comparable. Then

$$
\begin{aligned}
\Omega_{P, G}^{\omega}(n) & =\frac{1}{|G|} \sum_{g \in G} \Omega_{P_{g}}^{\omega_{g}}(n), \\
\Omega_{P, G}^{\omega}(-n) & =(-1)^{|P|}\left|\operatorname{Hom}_{+}^{-\omega}(P,[n]) / G\right| .
\end{aligned}
$$

\section{Graphs}

Let $\Gamma=(V, E)$ be a finite simple graph and let $G$ be a finite group acting on $\Gamma$ by automorphisms. A $\boldsymbol{n}$-coloring of $\Gamma$ is a map $c: V \rightarrow[n]$. The coloring is called proper if $c(v) \neq c(w)$ whenever there is an edge between $v$ and $w$. The action of $G$ on $\Gamma$ induces an action on the set of all colorings, and also on the set of all proper colorings which we denote by $\operatorname{Col}_{n}(\Gamma)$. The orbital chromatic polynomial $\chi_{\Gamma, G}$ is defined by $\chi_{\Gamma, G}(n)=\left|\operatorname{Col}_{n}(\Gamma) / G\right|$ for all $n \geqslant 1$. An orientation $\sigma: E \rightarrow V$ of $\Gamma$ assigns to every edge $e$ a vertex of $e$ called its head. An orientation is acyclic if there are no directed cycles. Every acyclic orientation $\sigma$ induces a partial order on the vertex set of $\Gamma$ by defining $v \prec_{\sigma} w$ if there is a directed path from $v$ to $w$. For the corresponding poset we write $\Gamma^{\sigma}$. $G$ acts on the set $\Sigma$ of all acyclic orientations of $\Gamma$ : For an edge $u v$ we define $(g \cdot \sigma)(u v)=g \cdot \sigma\left(g^{-1} \cdot u v\right)$. The next theorem gives us an expression of $\chi_{\Gamma, G}(n)$ in terms of order polynomials. In particular, $\chi_{\Gamma, G}(n)$ is a polynomial for all $n \geqslant 1$.

Theorem 3.1. Let $\Gamma$ be a graph and let $G$ be a group acting on $\Gamma$. Then $G$ acts on $\mathrm{Col}_{n}(\Gamma)$ and we have

$$
\chi_{\Gamma, G}(n)=\frac{1}{|G|} \sum_{g \in G} \sum_{\sigma \in \Sigma^{g}} \Omega_{\Gamma_{g}^{\sigma}}^{\circ}(n)
$$

for all $n \geqslant 1$. In particular, $\chi_{\Gamma, G}(n)$ agrees with a polynomial of degree $|\Gamma|$ for all $n \geqslant 1$.

Proof. By Theorem 2.1 we have

$$
\left|\operatorname{Col}_{n}(\Gamma) / G\right|=\frac{1}{|G|} \sum_{g \in G}\left|\operatorname{Col}_{n}(\Gamma)^{g}\right|
$$

Let $\phi$ be an element of $\operatorname{Col}_{n}(\Gamma)^{g}$ and let $\sigma$ be the acyclic orientation induced by the coloring $\phi$, i.e. an edge $e=u v$ is oriented from $u$ to $v$ whenever $\phi(u)<\phi(v)$. Then $\phi$ is a 
strictly order preserving map from $\Gamma^{\sigma}$ into $[n]$ and $\sigma$ is fixed by $g$, because for every edge $v w \in E$ we have $v \prec_{\sigma} w$ by definition if and only if $\phi(v)<\phi(w)$, and as $\phi$ is fixed by $g$, this implies $\phi(g v)<\phi(g w)$ which is equivalent to $g v \prec_{\sigma} g w$, i.e. $\sigma \in \Sigma^{g}$.

Example 3.2. Let $k>2$. We consider a cycle $C^{k}$ on $k$ vertices $\left\{x_{i}\right\}_{i \in \mathbb{Z}_{k}}$. Then its symmetry group is the dihedral group

$$
D_{k}=\left\langle r, s \mid r^{k}=1, s^{2}=1, s r s^{-1}=r^{-1}\right\rangle
$$

which acts on $C^{k}$ by

$$
\begin{aligned}
& r \cdot x_{i}=x_{i+1} \\
& s \cdot x_{i}=x_{-i}
\end{aligned}
$$

Then

$$
\chi_{C^{k}, D_{k}}(n)=\frac{1}{2 k}\left(\sum_{l=1}^{k}\left|\operatorname{Col}_{n}\left(C^{k}\right)^{r^{l}}\right|+\sum_{l=1}^{k}\left|\operatorname{Col}_{n}\left(C^{k}\right)^{s r^{l}}\right|\right) .
$$

Let $c \in \operatorname{Col}_{n}\left(C^{k}\right)$. If $l=2 q$ is even, then $s r^{2 q} \cdot c=c \Leftrightarrow\left(r^{q} \cdot c\right)=s \cdot\left(r^{q} \cdot c\right)$ and therefore

$$
\left|\operatorname{Col}_{n}\left(C^{k}\right)^{s r^{l}}\right|=\left|\operatorname{Col}_{n}\left(C^{k}\right)^{s}\right|= \begin{cases}\left|\operatorname{Col}_{n}\left(\left[\frac{k}{2}+1\right]\right)\right| & \text { if } k \text { is even } \\ 0 & \text { otherwise. }\end{cases}
$$

If $l=2 q+1$ is odd, then $s r^{2 q+1} \cdot c=c \Leftrightarrow\left(r^{q+1} \cdot c\right)=r s \cdot\left(r^{q+1} \cdot c\right)$ and therefore

$$
\left|\operatorname{Col}_{n}\left(C^{k}\right)^{s \cdot r^{l}}\right|=\left|\operatorname{Col}_{n}\left(C^{k}\right)^{r s}\right|=0
$$

as $r s \cdot x_{0}=x_{1}$, and $x_{0}$ and $x_{1}$ are connected by an edge. Further, for all $1 \leqslant l \leqslant k$ we obtain

$$
\left|\operatorname{Col}_{n}\left(C^{k}\right)^{r^{l}}\right|= \begin{cases}\left|\operatorname{Col}_{n}\left(C^{m}\right)\right| & \text { if } m=\operatorname{gcd}(l, k) \neq 1, \\ 0 & \text { otherwise. }\end{cases}
$$

If $k>1$ is odd, we therefore get

$$
\chi_{C^{k}, D_{k}}(n)=\frac{1}{2} \chi_{C^{k}, \mathbb{Z}_{k}}(n),
$$

with $\mathbb{Z}_{k}:=\mathbb{Z} / k \mathbb{Z}=\langle r\rangle \subset D_{k}$. If $k=p>2$ is a prime this simplifies even further:

$$
\left|\mathrm{Col}_{n}\left(C^{p}\right) / D_{p}\right|=\frac{1}{2 p}\left|\operatorname{Col}_{n}\left(C^{p}\right)\right| .
$$

This is reminiscient of counting necklaces with colored beads (see e.g. [9, Chapter 35]). 
A pair $(c, \sigma)$ consisting of a coloring $c: V \rightarrow[n]$ and an acyclic orientation $\sigma: E \rightarrow V$ is called weakly compatible if for every edge $e=u v$ we have $\sigma(u v)=v$ whenever $c(u)<c(v)$. We define

$$
\Sigma_{n}(\Gamma)=\left\{(c, \sigma) \in[n]^{V} \times \Sigma: \text { weakly compatible }\right\}
$$

If $G$ acts on $\Gamma$ by automorphisms it also acts on $\Sigma_{n}(\Gamma)$ by $g \cdot(c, \sigma)=(g \cdot c, g \cdot \sigma)$ for all $(c, \sigma) \in \Sigma_{n}(\Gamma)$ and $g \in G$. An element $(c, \sigma) \in \Sigma_{n}(\Gamma)$ is called even if for all $g \in \operatorname{Stab}((c, \sigma))$ we have $\operatorname{sgn}(g)=1$ as permutation of the vertices. We denote the set of all even elements of $\Sigma_{n}(\Gamma)$ by $\Sigma_{n,+}(\Gamma)$. The action of $G$ restricts to an action on $\Sigma_{n,+}(\Gamma)$. We get the following reciprocity statement:

Theorem 3.3. Let $\Gamma$ be a graph and $G$ a group acting on $\Gamma$. Then

$$
\chi_{\Gamma, G}(-n)=(-1)^{|\Gamma|}\left|\Sigma_{n,+}(\Gamma) / G\right|
$$

Proof. By Theorem 3.1 and Theorem 2.3 and $\operatorname{sgn}(g)=(-1)^{|\Gamma|+c(g)}$ we have

$$
\chi_{\Gamma, G}(-n)=(-1)^{|\Gamma|} \frac{1}{|G|} \sum_{g \in G} \operatorname{sgn}(g) \sum_{\sigma \in \Sigma^{g}} \Omega_{\Gamma_{g}^{\sigma}}(n) .
$$

As in the proof of Theorem 2.5 we see $\Omega_{\Gamma_{g}^{\sigma}}(n)=\left|\operatorname{Hom}\left(\Gamma^{\sigma},[n]\right)^{g}\right|$, and we observe

$$
\left|\Sigma_{n}(\Gamma)^{g}\right|=\sum_{\sigma \in \Sigma^{g}}\left|\operatorname{Hom}\left(\Gamma^{\sigma},[n]\right)^{g}\right| .
$$

Now we argue the same way as in the proof of Theorem 2.8: By equation (6) we get

$$
\chi_{\Gamma, G}(-n)=(-1)^{|\Gamma|} \frac{1}{|G|} \sum_{g \in G} \operatorname{sgn} g\left|\Sigma_{n}(\Gamma)^{g}\right|=(-1)^{|\Gamma|} \frac{1}{|G|} \sum_{(c, \sigma) \in \Sigma_{n}(\Gamma)} \sum_{g \in \operatorname{Stab}((c, \sigma))} \operatorname{sgn}(g) .
$$

For $(c, \sigma) \in \Sigma_{n}(\Gamma)$ and $g_{0} \in \operatorname{Stab}((c, \sigma))$ such that $\operatorname{sgn} g_{0}=-1$ as permutation of the vertices there is a bijection

$$
\begin{aligned}
\{g \in \operatorname{Stab}((c, \sigma)): \operatorname{sgn}(g)=1\} & \longrightarrow\{g \in \operatorname{Stab}((c, \sigma)): \operatorname{sgn}(g)=-1\} \\
g & \mapsto g_{0} g
\end{aligned}
$$

Hence, $\sum_{g \in \operatorname{Stab}((c, \sigma))} \operatorname{sgn}(g)=0$ whenever $(c, \sigma)$ is not even. Therefore the right hand side of equation (7) equals

$$
(-1)^{|\Gamma|} \frac{1}{|G|} \sum_{g \in G}\left|\Sigma_{n,+}(\Gamma)^{g}\right|
$$

which by Theorem 2.1 equals $(-1)^{|\Gamma|}\left|\Sigma_{n,+}(\Gamma) / G\right|$.

An easy interpretation can be given in the case of $G=\mathbb{Z}_{2}$ : 
Corollary 3.4. Let $\Gamma$ be a graph and let $\mathbb{Z}_{2}=\{e, \tau\}$ act on $\Gamma$ by automorphisms such that $\operatorname{sgn} \tau=-1$. Then

$$
\chi_{\Gamma, \mathbb{Z}_{2}}(-1)=(-1)^{|\Gamma|} \frac{\left|\Sigma_{+}\right|}{2}
$$

where $\Sigma_{+}=\Sigma_{1,+}(\Gamma)$ is the set of even acyclic orientations of $\Gamma$.

For $G$ acting trivially on $\Gamma$ we recover a well-known theorem by Stanley:

Corollary 3.5 ([6, Thm. 1.2]). Let $\Gamma$ be a graph and $\chi_{\Gamma}$ its chromatic polynomial. Then $\left|\chi_{\Gamma}(-n)\right|$ equals the number of weakly compatible pairs $(c, \sigma)$ consisting of a $n$-coloring $c$ and an acyclic orientation $\sigma$ of $\Gamma$. In particular, $\left|\chi_{\Gamma}(-1)\right|$ is the number of acyclic orientations of $\Gamma$.

Similarly as in Theorem 2.8 there is a twin reciprocity in the case of graph colorings. We say that a $n$-coloring $c$ of $\Gamma$ is even if for all $g \in \operatorname{Stab}(c)$ we have $\operatorname{sgn} g=1$ and define $\mathrm{Col}_{n,+}(\Gamma)$ as the set of all even proper $n$-colorings of $\Gamma$. Then the action of $G$ on $\operatorname{Col}_{n}(\Gamma)$ restricts to an action on $\operatorname{Col}_{n,+}(\Gamma)$. We further define $\chi_{\Gamma, G}^{+}(n)=\left|\mathrm{Col}_{n,+}(\Gamma) / G\right|$ as the function counting the number of orbits of even proper $n$-colorings for $n \geqslant 1$. By similar arguments as in Theorem 3.1 and Theorem 3.3 we then have the following:

Proposition 3.6. Let $\Gamma$ be a graph and $G$ a group acting on $\Gamma$ by automorphisms. Then $\chi_{\Gamma, G}^{+}(n)$ agrees with a polynomial of degree $|\Gamma|$ for $n \geqslant 1$ and we have

$$
\chi_{\Gamma, G}^{+}(-n)=(-1)^{|\Gamma|}\left|\Sigma_{n}(\Gamma) / G\right| .
$$

\section{Acknowledgments}

We are grateful to Raman Sanyal for suggesting this project and constant support. We also want to thank Alexandru Chirvasitu and Yan X. Zhang for fruitful conversations, and Darij Grinberg for careful reading and helpful comments. We are furthermore very thankful to the anonymous referee for suggesting Theorem 2.13.

\section{References}

[1] P. J. Cameron and K. K. Kayibi, Orbital chromatic and flow roots, Combinatorics, Probability and Computing, 16 (2007), pp. 401-407.

[2] P. Hanlon, The chromatic polynomial of an unlabeled graph, Journal of Combinatorial Theory, Series B, 38 (1985), pp. 226-239.

[3] G. PÓLYA, Kombinatorische Anzahlbestimmungen für Gruppen, Graphen und chemische Verbindungen, Acta mathematica, 68 (1937), pp. 145-254.

[4] R. P. Stanley, A chromatic-like polynomial for ordered sets, in Proceedings of the Second Chapel Hill Conference on Combinatorial Mathematics and its Applications, 1970, pp. 421-427. 
[5] _ - Ordered structures and partitions, vol. 119, American Mathematical Soc., 1972.

[6] __, Acyclic orientations of graphs, Discrete Mathematics, 5 (1973), pp. 171-178.

[7] —, Quotients of Peck posets, Order, 1 (1984), pp. 29-34.

[8] A. Stapledon, Equivariant Ehrhart theory, Advances in Mathematics, 226 (2011), pp. 3622-3654.

[9] J. H. van Lint and R. M. Wilson, A course in combinatorics, Cambridge University Press, Cambridge, second ed., 2001. 